\title{
Strategic Directions in Tissue Engineering
}

\author{
PETER C. JOHNSON, M.D., ${ }^{1}$ ANTONIOS G. MIKOS, Ph.D., ${ }^{2}$ \\ JOHN P. FISHER, Ph.D., ${ }^{3}$ and JOHN A. JANSEN, D.D.S., Ph.D. ${ }^{4}$
}

\begin{abstract}
The field of tissue engineering is developing rapidly. Given its ultimate importance to clinical care, the time is appropriate to assess the field's strategic directions to optimize research and development activities. To characterize strategic directions in tissue engineering, a distant but reachable clinical goal was proposed and a worldwide body of 24 leaders in tissue engineering was queried systematically to determine the best paths toward that goal. Using a modified Hoshin process, we identified 14 critical activity categories and then stratified them by their immediate priority for the field. The result of the analysis illustrates a highly interdependent set of activities that are dominated by the need for an understanding of angiogenesis, stem cell science, and the utilization of molecular biology and systems biology tools to enable a deeper comprehension of tissue development and control.
\end{abstract}

\section{INTRODUCTION}

$\mathbf{T}$ HE FIELD OF TISSUE ENGINEERING IS DIVERSE in its methods and clinical targets and holds great promise for improved patient care. It has matured to the point that its activities can be reasonably well categorized, providing the opportunity for a strategic assessment that is able to guide researchers, funders, policy makers, regulators, and technology developers. We undertook such an analysis with the participation of the international editorial board of the journal. Our hope was to obtain a strategic perspective on the field from the point of view of those who are actively developing its technologies around the world. In particular, we concentrated on three topics:

1. Determining the critical activity foci for the field that will enable it to progress

2. Characterizing the degree to which we have made progress in these areas

3. Determining the linkages between activities and stratify them on the basis of their predominance as influencers of one another while taking into consideration previous progress
In this way, we intended to provide ourselves as editors with direction regarding the most critical issues facing the field. In addition, we wanted to present the community with the experienced perspective that our worldwide editorial board has provided with respect to these issues.

To achieve this, a modified Hoshin* facilitation technique was used that uniquely enabled asynchronous international participation to complete the assessment. Members of the editorial board were asked to identify critical steps that would be needed to achieve the following goal: "The field of tissue engineering will exhibit broad clinical success by the year 2021."

Based upon their responses, a list of strategically important concepts was identified and prioritized on basis of their immediate importance to the field. The data obtained (listed in Appendix A) provide a focus for research and development activities in the field that may assist in the

*Hoshin method-also known as the Hoshin Kanri method that means "sharp pointed metal" like the needle of a compass-is a strategic planning methodology that defines a mission, and then identifies and prioritizes the tasks needed to achieve the mission.

\footnotetext{
${ }^{1}$ Scintellix, LLC, Raleigh, North Carolina.

${ }^{2}$ Department of Bioengineering, Rice University, Houston, Texas.

${ }^{3}$ Fischell Department of Bioengineering, University of Maryland, College Park, Maryland.

${ }^{4}$ Department of Periodontology and Biomaterials, Radboud University Nijmegen Medical Center, Nijmegen, The Netherlands.
} 
enhancement of research and development efficiency and financing.

\section{MATERIALS AND METHODS}

\section{Participants in the assessment}

Twenty-four members of the editorial board of the journal Tissue Engineering participated in this assessment. (They are listed in the "Acknowledgments" section.)

\section{Modified Hoshin method}

The Hoshin facilitation process is a well-established strategic planning technique. It is designed to support group strategic planning processes through the identification of a common goal followed by delineation and prioritization of the steps needed to achieve the goal. It is especially effective in disparate groups, as it is designed to maximize the sharing of ideas through active group participation. Although the Hoshin process is normally run with a group working together at one location in real time, the nature of our participant community did not allow this. In this modified approach, one set of participants (the editorial board members) was guided asynchronously through one part of the process while the four editors-in-chief acted synchronously in the assessment of the ideas provided by the editorial board.

The steps of the modified Hoshin process were as follows:

1. Mission creation: The mission (see above) was selected and communicated electronically to the editorial board. When the ideas were collected in late 2006, the goal was 15 years distant. This duration was felt to credibly estimate the time needed for the field to express significant clinical development potential.

2. Idea generation: The editorial board members were asked to provide up to 10 critical ideas or steps that would need to occur for the mission to be successful. Twenty-four editorial board members representing Asia, Africa, Europe, and North America participated in the response.

3. Electronic idea collection: The ideas (145) were collected from the editorial board members by e-mail.

4. Clustering of related ideas: Once all ideas were collected, they were sorted on the basis of their similarity into 14 distinct groups of ideas (see Appendix A).

5. Naming of clusters as concepts: The clustered idea groups were provided an overarching name that encompassed the content of its collective ideas. These clusters, known as "concepts," are, in essence, activity foci for the field.

6. Comparison of concepts to determine raw dominance: Using standard Hoshin approaches, the concepts were placed in a circle on paper and compared to one another in sequential fashion until all pairwise comparisons were completed. In each instance, the question was asked: "Is there a relationship between these concepts?" If so, a line was drawn between the concepts and a unidirectional arrow was assigned leading from the dominant concept to the one that was dominated. Once complete, the number of relationships $(R)$ and the number of "out" or "dominant" arrows $(O)$ were counted. This process was performed by the editors-in-chief.

7. Assessment of progress to date: Each editor-in-chief assigned a level of progress achieved thus far to each concept. A scale of $1=$ no progress to $10=$ complete progress was assigned. The mean progress level $(P)$ assigned by the four editors was used as the concept progress designation for each concept.

8. Determination of normalized concept dominance: To determine the most important concepts for immediate focus, the dominance $(O)$ of each concept was divided by its level of present progress $(P)$ and the divisor $O / P$ was obtained for each. On the basis of this figure, concepts were sorted from most to least dominant.

\section{RESULTS}

\section{Distillation of concepts from raw ideas}

The 145 raw ideas provided by members of the editorial board were grouped into concept categories on the basis of their relatedness. Fourteen concepts were derived in this way, as shown in Table 1. The similar ideas that led to each concept classification are shown in the table, and the full text of these ideas is provided in Appendix A.

\section{Raw Hoshin data}

Table 2 presents the raw data from the Hoshin analysis, including the number of outgoing (dominant) arrows $(O)$, the total number of relationships of each concept with the others $(R)$, the mean estimated progress in the field thus far $(P)$, and the dividend of $O / P$, the dominance of the concept

Table 1. Concepts and Numbers of Raw Ideas Supporting Them

Clinical understanding/interaction $\quad 21$

Manufacturing/scale-up $\quad 19$

Cell sourcing and cell/tissue characterization $\quad 15$

Molecular biology/systems biology 15

$\begin{array}{ll}\text { Multilevel funding } & 12\end{array}$

Stem cell science $\quad 11$

Pharmacoeconomic/commercial pathway 9

Enhanced biomaterial functionality $\quad 8$

Standardized models $\quad 8$

$\begin{array}{ll}\text { Regulatory transparency } & 7\end{array}$

Multidisciplinary understanding/cooperation 6

Immunologic understanding and control 5

Expectation management/communication $\quad 5$

Angiogenic control 4 
Table 2. Raw Hoshin Data

\begin{tabular}{|c|c|c|c|c|}
\hline & \multicolumn{4}{|c|}{ Mean } \\
\hline & $O$ & $R$ & Mean $P$ & $O / P$ \\
\hline Clinical understanding/interaction & 10 & 13 & 4.5 & 2.2 \\
\hline Manufacturing/scale-up & 4 & 11 & 3.5 & 1.1 \\
\hline Cell sourcing and cell/tissue characterization & 10 & 12 & 3.8 & 2.7 \\
\hline Molecular biology/systems biology & 11 & 11 & 4.0 & 2.8 \\
\hline Multilevel funding & 0 & 13 & 4.8 & 0.0 \\
\hline Stem cell science & 12 & 13 & 3.8 & 3.2 \\
\hline Pharmacoeconomic/commercial pathway & 1 & 12 & 3.8 & 0.3 \\
\hline Enhanced biomaterial functionality & 4 & 13 & 4.8 & 0.8 \\
\hline Standardized models & 5 & 11 & 4.8 & 1.1 \\
\hline Regulatory transparency & 5 & 13 & 4.5 & 1.1 \\
\hline Multidisciplinary understanding/cooperation & 5 & 13 & 6.5 & 0.8 \\
\hline Immunologic understanding and control & 7 & 12 & 3.5 & 2.0 \\
\hline Expectation management/communication & 2 & 10 & 5.5 & 0.4 \\
\hline Angiogenic control & 9 & 13 & 2.8 & 3.3 \\
\hline Mean & 6.1 & 12.1 & 4.3 & 1.5 \\
\hline SD & 3.8 & 1.0 & 0.9 & 1.1 \\
\hline
\end{tabular}

normalized to present progress. The mean number of dominant relationships per category was $6.1 \pm 3.8$. The mean number of relationships each concept had (out of a possible 13) was $12.1 \pm 1.0$. The mean level of progress in the field per concept was $4.3 \pm 0.9$ out of a possible 10 .

\section{Concept relationship strength}

As shown in Table 3, when sorted on the basis of relationship strength per category, the scattering of data is very small, indicating an intense interrelatedness of critical concepts in the field.

\section{Raw dominance of concepts over one another}

In Table 4, the concepts are depicted in the order of decreasing strength over one another. This listing represents

Table 3. Concepts Sorted as a Function of Relationship STRENGTH

\begin{tabular}{ll}
\hline Clinical understanding/interaction & 13 \\
Multilevel funding & 13 \\
Stem cell science & 13 \\
Enhanced biomaterial functionality & 13 \\
Regulatory transparency & 13 \\
Multidisciplinary understanding/cooperation & 13 \\
Angiogenic control & 13 \\
Cell sourcing and cell/tissue characterization & 12 \\
Pharmacoeconomic/commercial pathway & 12 \\
Immunologic understanding and control & 12 \\
Manufacturing/scale-up & 11 \\
Molecular biology/systems biology & 11 \\
Standardized models & 11 \\
Expectation management/communication & 10 \\
\hline
\end{tabular}

the raw importance of the concepts to the field without taking into consideration progress to this point.

\section{Concept progress thus far}

Table 5 shows the estimated progress achieved thus far ( $1=$ none; $10=$ complete $)$ for each concept.

\section{Normalized concept dominance (i.e., when} progress has been taken into consideration)

Table 6 illustrates the final dominance profile of the concept areas once present levels of progress have been taken into consideration. This is the sorted order of the most critical concepts that require pursuit at this time.

\section{Table 4. Concepts Sorted by Raw Dominance}

\section{Stem cell science} 12 Molecular biology/systems biology 11 Clinical understanding/interaction 10

Cell sourcing and cell/tissue characterization 10 Angiogenic control

Immunologic understanding and control

Standardized models

Regulatory transparency

Multidisciplinary understanding/cooperation

Manufacturing/scale-up

Enhanced biomaterial functionality

Expectation management/communication

Pharmacoeconomic/commercial pathway

Multilevel funding

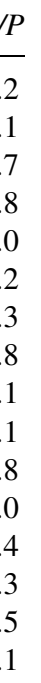


Table 5. Concepts Sorted as a Function of Progress Achieved Thus Far

\begin{tabular}{lc}
\hline & $P$ \\
\hline Multidisciplinary understanding/cooperation & 6.5 \\
Expectation management/communication & 5.5 \\
Multilevel funding & 4.8 \\
Enhanced biomaterial functionality & 4.8 \\
Standardized models & 4.8 \\
Clinical understanding/interaction & 4.5 \\
Regulatory transparency & 4.5 \\
Molecular biology/systems biology & 4.0 \\
Cell sourcing and cell/tissue characterization & 3.8 \\
Stem cell science & 3.8 \\
Pharmacoeconomic/commercial pathway & 3.8 \\
Manufacturing/scale-up & 3.5 \\
Immunologic understanding and control & 3.5 \\
Angiogenic control & 2.8 \\
\hline
\end{tabular}

\section{Interconcept relationships}

Table 7 reflects the actual relationships between concepts. For each concept (lettered on the left), the group of other concepts it dominates is shown on the right.

\section{DISCUSSION}

A check on the strategic direction of a field as diverse as tissue engineering by its practitioners is periodically warranted, if only to help focus our collective international strengths. We originally performed this analysis to identify key focus areas for invited reviews and editorials for the journal Tissue Engineering. However, as its process unfolded, it became clear that since the analysis incorporated the thoughts of many of the world's leaders in tissue

Table 6. Normalized Concept Dominance (i.e., Taking Present Progress into Consideration)

\begin{tabular}{lc}
\hline & $O / P$ \\
\hline Angiogenic control & 3.3 \\
Stem cell science & 3.2 \\
Molecular biology/systems biology & 2.8 \\
Cell sourcing and cell/tissue characterization & 2.7 \\
Clinical understanding/interaction & 2.2 \\
Immunologic understanding and control & 2.0 \\
Manufacturing/scale-up & 1.1 \\
Regulatory transparency & 1.1 \\
Standardized models & 1.1 \\
Enhanced biomaterial functionality & 0.8 \\
Multidisciplinary understanding/cooperation & 0.8 \\
Expectation management/communication & 0.4 \\
Pharmacoeconomic/commercial pathway & 0.3 \\
Multilevel funding & 0.0 \\
\hline
\end{tabular}

engineering, it might have a broader role in guiding the direction of development of the field.

Since tissue engineering is ultimately focused on the improvement of the care of patients, we chose a relevant goal - the clinical success of the field by 2021 — and then culled the opinions of 24 key international leaders to determine the critical steps en route to this goal. They independently created and submitted their responses, providing a thoughtful snapshot of international opinion regarding strategic directions in the field. Using the Hoshin analysis methodology, these contributions were grouped by similarity, classified into concepts, and the concepts were then compared by the editors-in-chief of Tissue Engineering Parts $\mathrm{A}, \mathrm{B}$, and $\mathrm{C}$ to determine their relative priority in support of progress in the field. The Hoshin process has the unique capacity to identify interrelationships between important strategic activity subsets. In this case, its application led to a deeper understanding of what constitute the most dominant directions in the field. Because of the way that concept areas are linked together, a focus on the most dominant concepts inevitably should lead to all concepts, in turn.

An important step in this analysis is the assignment of present progress in the field. While this is a subjective assessment, it has proven itself to be a helpful tool in Hoshin analyses since it allows very dominant ideas to be normalized by the degree to which previous progress has been made. Periodic progress reassessment and analysis of the effect on concept dominance will be important in the years ahead, to restructure priorities for the attention of funding agencies and the like.

In the process of performing the analysis, we learned several things. While some concept areas received attention from many of the responding board members, the number of ideas a concept area received did not correlate with its ultimate strategic importance. This suggests that highly strategic issues often may not lie at the forefront of our day-to-day conception of the most important foci in the field, making such analyses more important to undertake. For example, the strategically most important category, angiogenic control, was supported by only four contributed ideas. However, its dominance over nine other concepts and its low level of present progress propelled it to the top of the list of strategic concepts. Clearly, mastering the control of angiogenesis will be at the heart of any attempts to grow larger tissueengineered constructs than have thus far been achievable. This will apply whether such growth occurs in vitro or within the body as a response to cell and/or scaffold implantation.

Stem cell science is the second most strategic concept, dominating 12 other concepts. It may well be that the understanding and control of stem cell development will enable us to short circuit some of the tissue engineering methods used heretofore-perhaps allowing the concurrent growth of vascular systems with parenchymal tissues.

To understand and control stem cell behavior, we need to be able to measure cellular responses at the molecular level 
Table 7. The Dominant Relationships between Concepts

\begin{tabular}{lll}
\hline A & Clinical understanding/interaction & B, E, G, H, I, J, K, L, M, N \\
B & Manufacturing/scale-up & E, G, H, M \\
C & Cell sourcing and cell/tissue characterization & A, B, E, G, H, I, J, K, L, N \\
D & Molecular biology/systems biology & A, B, C, E, F, H, I, J, K, L, N \\
E & Multilevel funding & \\
F & Stem cell science & A, B, C, E, G, H, I, J, K, L, M, N \\
G & Pharmacoeconomic/commercial pathway & E \\
H & Enhanced biomaterial functionality & E, G, K, M \\
I & Standardized models & E, G, H, J, K \\
J & Regulatory transparency & B, E, G, H, M \\
K & Multidisciplinary understanding/cooperation & B, E, G, J, M \\
L & Immunologic understanding and control & E, G, H, I, J, K, M \\
M & Expectation management/communication & E, G \\
N & Angiogenic control & B, E, G, H, I, J, K, L, M \\
\hline
\end{tabular}

and thereby characterize cause-and-effect relationships. Recent advances in molecular biology and systems biology enable us to do this, making this concept area highly strategic for future developments throughout the field. The continuum of measurable cell characteristics (genome sequence, gene expression arrays, and proteomic and metabolomic patterns) can now be combined with digital automated histology information to provide a systems biology view of tissue development. Information systems are now capable of properly aggregating and analyzing such information, enabling the identification of biomarkers of development that can be used for guidance in engineered tissue development.

While standardized cell sourcing for tissue engineering applications remains a problem in flux, the characterization of cells and tissues, as stated above, will make whatever cells that are sourced be far better understood in their ultimate behaviors. Cell sourcing remains a critically important focus area for the field.

At this point in the analysis, the concept that was supported by the largest number of ideas, clinical understanding and interaction, finds its strategic position. Clearly, if the field is oriented toward clinical applications, close engagement of the clinical community is important. This is not only valuable for the establishment of engineered tissue design criteria but also to enhance the potential for the ultimate acceptance of such therapies into clinical practice at large. Fortunately, significant progress has been made in the weaving of scientific and engineering groups together with clinicians for the mutual development of the field.

Though often overlooked, immunologic understanding and control will be an important hurdle for the field in the coming years. Depending upon the sources of standardized cells (autologous vs. allogeneic) and on the antigen presentation of cells derived from stem cells, immunologic understanding will prove to be of increasing importance. The development of a discipline known as the Immunology of Engineered Tissue would be timely, so that the need for immunologic response controls in the face of implanted engineered tissues can be understood.
Though perhaps premature in some cases, the ultimate need for manufacturing/scale-up will be prevalent throughout the field. It is placed high on the list of strategic concepts for this reason. Issues in manufacturing and scale-up go hand in hand with those of cell sourcing and characterization. Several groups around the world are formally engaged in this discipline at this time.

Regulatory transparency is next on the strategic concept list. While a great deal of effort has been expended by United States and international regulatory authorities in preparing to understand and regulate engineered tissues, clear pathways through the regulatory process have not yet been completely defined. It is supposed that the greater capacity for cell and tissue characterization using molecular biology and systems biology tools will provide assurances regarding cell behavior and fate that will enable regulatory authorities to assess the appropriate data needed to transparently regulate tissueengineered products.

Regulatory authorities will benefit from the development of acceptable standardized models of engineered tissue implantation. It is early in the process of developing such models but they will be critically needed as the next wave of engineered tissue technologies reaches preclinical experimentation milestones.

While biomaterial scaffolds have been a mainstay in the field for years, the strategic importance of enhanced biomaterial functionality depends quite heavily on what we learn about cells and tissues using the new characterization methodologies. While functional biomaterials will be of great importance as delivery vehicles for engineered tissue technologies, their design will grow from the known natural needs and responses of functional tissues themselves.

Fortunately, substantial progress has been made in the concept area of multidisciplinary understanding and cooperation. Indeed, this is something of a birthright for the field of tissue engineering, which put so much effort into this focal area in its early years, not only within research groups but also through the many regional initiatives that have dotted the landscape of the field. Collaboration and information sharing 
with an eye toward effective communication are among the pillars of the field's development.

Similarly, while the field initially perhaps touted its potential with excessive alacrity, we seem to have matured into a solid appreciation of the importance of expectation management and communication so that future advances can be received by clinicians and the public as having the real value stated at the time.

Commercial processes for the development of tissueengineered products are known to be critical for these products to reach the bedside. Fortunately, the analysis shows that knowledge of how these processes work is generally extant, making pharmacoeconomic/commercial pathway considerations more generally resolvable and therefore of less critical importance on the immediate strategic pathway. This may change as nascent technologies ripen and commercial pathway issues become rate limiting. This underscores the need for a periodic reassessment of strategic data.

Finally, while one might think reflexively that multilevel funding is the key driver of the field, in fact it appears to be the opposite. That is, funds are actually allocated and released at both research and commercialization levels on the basis of the credibility of technology or its potential for development. As a result, funding decisions are made on the basis of successful progress in other strategic concept areas, such as evidence of clinical and multidisciplinary interaction, availability of tools for cell and tissue characterization, harnessing of stem cell responses, and the like. While funding is a chicken-and-egg situation, it is clear from the beginnings of the field that substantial funding always follows tangible technology development.

When the analysis is viewed through the lens of progress achieved in the field thus far, it is clear that a good job has been done by bringing scientific, engineering, and clinical disciplines together, by enabling the generation of a substantial stream of funding, by moving forward the development of biomaterials, and by the creation of a reasonable regulatory transparency. However, a great deal of work has yet to be done before angiogenic systems can be created for advanced tissue growth, stem cell and immunologic behavior is tied together meaningfully, and all of our scale-up needs are reached. This is why the latter rate highly in the priority of strategic concept areas.

Fortunately, as we progress, comfort can be taken in the fact that developments in any of these areas are likely to positively impact the whole of the field. Remarkably, when the number of relationships was measured per concept area, the mean number of relationships per concept area (out of a possible 13) was 12.1, six of these being dominant relationships.

Recently, a strategic assessment of the field from the MATES group of Federal agencies was released. ${ }^{\dagger}$ The

\footnotetext{
${ }^{\dagger}$ Advancing Tissue Science and Engineering: A Multi-Agency Strategic Plan, U.S. Government Multi-Agency Tissue Engineering Science (MATES) Interagency Working Group, National Science and Technology Council, 2007. Web site: http://tissueengineering.gov/welcome-s.htm.
}

results of this multiyear project provided the following critical priorities for the field:

- "Understanding the Cellular Machinery

- Identifying, Validating Biomarkers and Assays

- Advancing Imaging Technologies

- Defining Cell/Environment Interactions

- Establishing Computational Modeling Systems

- Assembling and Maintaining Complex Tissue

- Improving Tissue Preservation and Storage

- Facilitating Effective Applications Development and Commercialization"

These were not prioritized but were listed in the order of the pathway from research to commercialization.

The report pointed out four overarching goals for the field:

1. "Understanding and controlling the cellular response: A fundamental challenge is to understand how cellsthe building blocks of tissues-receive and respond to information from their local environment in establishing and maintaining tissues.

2. Formulating biomaterial scaffolds and the tissue matrix environment: The scaffolding that supports cells and gives tissues their form is increasingly appreciated as an important source of information that drives cell fate determination. A deeper understanding of the biology underlying this relationship will allow more effective tissue design and engineering.

3. Developing enabling tools: Complex, multiparametric inputs are required to assess the state of a tissue and the cells within it. This information will be supplied by improvements in high-throughput assays and instrumentation, imaging modalities, fabrication technologies, computational modeling, and bioinformatics. Additionally, tissue preservation technologies and bioreactors will facilitate the generation of tissues on demand.

4. Promoting scale-up, translation, and commercialization: Demonstrating the feasibility of designing an engineered tissue is not enough. Realizing the full benefits tissue engineering science requires increased reproducibility, robustness, and user-friendliness that will enable the broad distribution of products."

In assessing worldwide opinion through our strategic planning process, it has become clear that the MATES group and the international community are thinking along similar lines. This is reassuring and exciting. It is our expectation that the progress assessment and prioritization provided by the Hoshin methodology, along with the tactical approaches suggested in the raw ideas (Appendix A), may add value within the overarching themes presented in the MATES report and also suggest some prioritization of funding support for the field. 
As editors of the Tissue Engineering journal, we anticipate that this analysis will be of some benefit to those planning their research, designing institutes and teaching programs, and allocating funding for the future development of the field.

\section{ACKNOWLEDGMENTS}

We would like to acknowledge the participation of the following members of the editorial board in the initial submission of ideas related to the overall goal: Stelios Andreadis, C. Mauli Agrawal, Anthony Atala, Francois Auger, Yilin Cao, Fu-Zhai Cui, Wouter Dhert, Timothy E. Hardingham, Ernst B. Hunziker, John A. Jansen, Peter C. Johnson, Hai Quan Mao, Jeremy Mao, Mona Marei, Ivan Martin, Chris Mason, Seeram Ramakrishna, Anthony Ratcliffe, Myron Spector, Bjoern Stark, Sing-Wen Sun, Bill Tawil, Joseph Vacanti, and Lee Weiss.

\section{APPENDIX A: CONCEPTS AND THEIR UNDERPINNING IDEAS}

The following list contains all the concept headings and the raw ideas they comprise. The ideas themselves provide good tactical directions for the enhancement of the field. They are listed here in unedited form to convey their initial meaning.

\section{Clinical Understanding/Interaction (21 ideas; 14.5\%)}

- Widespread buy-in by clinicians and healthcare providers (Market pull)

- Define by clinical epidemiology the area for needs for tissue engineering

- Define on a public health level the needs and the cost implications

- Consider that "engineering" requires a "design"

- Clinical trials. There need to be more (Phase 1) human trials of tissue-engineered constructs. This relates to the importance of employing relevant animal models in preclinical investigation.

- Close interactions between bioengineers and clinical scientists

- Get support for clinical trials

- Identification of achievable clinical targets (can it be done?)

- Focus research on clinically relevant issues (priority of clinic over technology)

- Engaging the clinical stakeholders more specifically the surgeons and trauma units

- Translation into the clinical arena for applied tissue engineering

- Centralized facilities versus decentralized facilities
- Encourage research on influence of boundary conditions on outcome of TE treatments (joint swelling, hematoma formation, diabetes, etc.)

- Successful carefully constructed and quantitated clinical trials associated with the successful creation of an International network

- Long-term patient/product follow-up registers (extension of Phase IV clinical trials)

- Shift resources from animal studies into professionally managed clinical trials, with validated outcome measures

- Demonstrate clear clinical success with a cell-based treatment of a significant disease (diabetes, Parkinson's, etc.)

- Development of strategies for clinical implementation of new TE treatments by identifying suitable patient (sub)populations

- Development of methodologies to translate measured patient-specific parameters into patient-specific therapies, i.e., there will not be silver-bullet, one therapy fits all solutions

- Identification of products that meet a clinical need (do not directly compete with products already on the market, meeting that need)

- Translation of proof-of-concept technology into actual clinical application and evaluation

- Build more complicated organs such as liver, kidney, and heart to overcome the shortage of these organs

\section{Manufacturing/Scale-Up (19 ideas; 13.1\%)}

- Scaling up of the tissue engineering products to the final dimensions necessary for the human patients

- Development of tissue regeneration acceleration technology

- Develop low-cost automated production of cell-based products

- Development of efficient manufacturing processes

- Scalable, cost-effective production under GMP (Good manufacturing practice)

- Develop closed bioreactor systems for standardized, safe, and controlled manufacture of grafts

- Reduce the cost of growing cells at the industrial level to encourage companies to build a central cell manufacturing sites

- Development of reproducible tissue regeneration methodology

- To be able to better store tissue-engineered products so they can last longer

- Simplicity and off-the-shelf products for ubiquitous use - Totally animal-free products with all components clearly defined and consistent in quality

- Identify quality controls for the implant potency (e.g., predictive markers of cell function, not just viability tests) 
- Develop low-cost automated production of cell-based products

- Development of workable tissue preservation technology

- Sharing safety/toxicity international databases

- Consistent robust products which can be stored for long periods and easily transported and implanted into patients

- To be able to better store tissue-engineered products so they can last longer

- In vitro tissue engineering for in vivo implantation

- Scale-up and bioreactors

- Development of appropriate animal models and preclinical evaluation criteria as indicators for clinical investigations

- Coculture of two or multiple types of cells/tissue

- Functional vascularization for large tissue

- New biodegradable materials with fine-tuned degradation and acceptable safety profiles for the development of combination products

- Characterization criteria for in vivo implantation

- Mass transport and mechanical (shear) effect on tissue development

- Cryopreservation of combination product

\section{Cell Sourcing and Cell/Tissue Characterization \\ (15 ideas; $7.3 \%$ )}

- Availability of distinct cell sources for tissue regeneration

- Cell and tissue characterization

- Adequate cell sourcing

- Cell characterization

- Better understanding the cellular, molecular \& structural components of healthy tissues. This information is needed to better build tissue engineering products.

- New imaging technology for postgrafting evaluation of tissue engineering substitutes

- Cell and tissue imaging

- Cell and tissue tracking

- Development of high-throughput methods for testing tissue function

- Development of universal donor cell lines

- Research studies including meaningful in vivo functionality studies

- Identification of a consistent and plentiful cell source

- A more thorough understanding of the biology and cell-cell interactions among different cell types

- Crystal ball

- Technologies to support cell therapies

- Human cell source, well characterized, standardized, serum free

- Materials and delivery routes for enhanced cell retention, coupling

- Challenges ahead

- Challenge 1: Human cell source

- Resident cells, stem cells, cell banks
- Cell culture without animal components

- Standardization of cell derivation \& characterization

Molecular Biology/Systems Biology (15 ideas; 7.3\%)

- Thorough control of the human genome of the cells involved

- Use of genomics and proteomic technologies to understand the structure/function relationship in engineered tissues

- A significantly deeper understanding must be gained about the biology/physiology of tissue regeneration at the genomic and proteomic levels

- Create a table of proteins that every healthy and diseased tissue (cells) secrete, i.e., create a profile. This information is needed to better build tissue engineering products

- Genetic profiling

- Development of regulatable gene delivery vectors to allow for manipulation of tissue function and engineering structure/function relationships

- Understanding cell differentiation in the context of three-dimensional tissues

- Better insight in cell-signaling pathways and development of new strategies for the usage of growth factors (including siRNA)

- Control of the microenvironment, signaling environment, mechanical environment, biological environment

- Establish procedures and protocols that ensure that stem cells and their progeny are well tolerated in allogeneic cell transplants

- Multiple growth factors and their roles to promote complete regeneration of tissue structures

- Signal regulation within ECMs

- Improved technologies for enabling safe and predictable biomimetic delivery of signaling molecules

- Understanding differentiation routes of human adult and/or human embryonic stem cells to one or more lineages

- Cell programming and developmental biology: Strategy for expansion of human embryonic stem cells under defined conditions

- Understanding of critical components of native stem cell niches

- Artificial stem cell niche with defined parameters

- Availability of new embryonic stem cell lines

- Continuous federal and private funding

- Corporate collaboration with academia

\section{Multilevel Funding (12 ideas; 8.3\%)}

- More funding of tissue engineering especially for projects with strong translational component

- Increased NIH funding for: 
- training graduates and post-docs to become the nextgeneration tissue engineers

- maintaining or expanding the academic research infrastructures in which they will training, including increased funding for R01s

- Sufficient capital investment from private and government sources

- P.S. I have four more bullets:

- More money

- More money

- More money

- More money :-)

- Investment at the early-mid level (post SBIR) stage

- Investment at the early (SBIR level) stage

- Availability of substantial R\&D financial support

- Early stage (R01) research being targeted at translational research with outcomes that can have impact

- NIH funding of translational research in tissue engineering, including large animal studies and integrative studies utilizing stem cells, biomaterials, and/or bioactive factors in an in vivo environment

- Sufficient corporate or venture capital investment

- Appropriate funding from venture capital and angel investors

- Investment both in more fundamental research and in more translational/clinical research

\section{Stem Cell Science (11 ideas; 7.6\%)}

- Understanding stem cell differentiation

- Develop strategies for endogenous regenerative therapies, i.e., using endogenous stem cells instead of ex vivo expanding cells

- Isolation, characterization of human adult stem cells from different adult tissues

- Pragmatic system of ethical regulation with respect to human embryonic stem cells and derived products

- Use of stem cells

- Stem cells (especially for allograft) for tissue regeneration

- Differentiation of stem cells (in vitro and in vivo)

- Robust, efficient, and predictable processes for expanding stem cell populations, in vitro, to sufficient numbers required for therapeutic delivery

- Increase fundamental knowledge of stem cell characterization, selection, and differentiation

- Route of delivery of stem cells for tissue regeneration

- Stem cell programming

- Understanding of signaling controls of fatespecification (tissue specific)

- Guided differentiation of embryonic stem cells and adult stem cells

- Genetic manipulation (stable transduction) of stem cells

- Topographical and biochemical cues controlling fate specification
- Biodegradable scaffolds for in vivo implantation as combination product with cells

- Maturation of stem cells or progenitor cells and integration in vivo

- Continuous federal and private funding

\section{Pharmacoeconomic/Commercial Pathway}

(9 ideas; 6.2\%)

- Identification of products that meet a clinical need (do not directly compete with products already on the market, meeting that need)

- Understanding the complete product development pathway for tissue engineering (not just the combination product FDA issue)

- Clear reimbursement guidelines

- Prove that TE solutions are more economical than traditional techniques

- Products which demonstrate very clear benefits over conventional products, i.e., a real step-change in patient therapy

- Analyze the commercial situation and do an appropriate market analysis to assure a long-term profitability for the motivation of industry

- Successful cost/benefit analysis of Tissue Engineering products WITH the input of tissue Engineering society and regenerative medicine societies

- Established international markets and distribution networks

- Stable global industry with a good supply of skilled labor and other resources including capital

\section{Enhanced Biomaterial Functionality (8 ideas; 5.5\%)}

- Scaffolding technology

- Spatial and temporal control of biochemical cues

- Local presentation of growth factors (sustained release and surface clustered)

- Cell selective interface and coculture capability

- Biomimetic functionalization of scaffold

- Stimuli-sensitive and injectable hydrogels

- Scaffold processing and relevant nanotechnology

- New generation of scaffolds

- Biomaterial characterization

- Proper design of scaffolds with "smart properties"

- Cell/gene and material interactions

- Engineering cell-cell interactions in 3D to mimic native tissues

- Inductive bone implants with the addition of either growth factors or expanded cells

- Challenge: Enabling technologies

- Cell instructive environments (scaffolds, bioreactors), functional imaging

- Vascularization (functional perfusion; capillary bed for oxygen supply)

- Rigorous animal models, imaging 


\section{Standardized Models (8 ideas; 5.5\%)}

- Standardized in vivo models

- Meaningful animal models

- Tools for more efficient discovery, e.g., how to efficiently (cost and time) search massive design spaces (in vitro and in vivo), and how to more efficiently utilize animal models

- Development/utilization of animal models that represent clinical problems

- Development of appropriate animal models (other than mice or rats) for testing of tissue-engineered products

- In vivo test in large animals using a clinically relevant model

- Controllable models for biological and medical research

- High-throughput screening, biomimetic context

- Tissue models of development, disease

- Implementation of animal models that relate directly to specific compelling applications of tissue engineering in the clinic. For example, many in vivo studies address strategies for the engineering of a selected tissue, but few investigate the tissue-engineered constructs in a model that closely reflects a specific clinical application, with the current "gold standards" for treating the disorder as controls.

\section{Regulatory Transparency (7 ideas; $4.8 \%$ )}

- Clear regulatory pathways with regulation achievable in a timely and cost-effective manner

- Regulatory expertise

- Clarify FDA regulatory guidelines for tissue engineering products

- Clarity from the FDA with respect to regulation of tissue regeneration products

- Obtain international consensus on the regulation of cell-based products

- Continuous dialogue with specialists within registration authorities as "partners" rather than judges

- Establish softer trial rules for "non commercial" clinical trials

\section{Multidisciplinary Understanding/Cooperation \\ (6 ideas; $4.1 \%$ )}

- Encourage the formation of consortia that work on a specific topic (low back pain, joint trauma, etc.)

- Bring different groups into one specific area vs. one group mastering all fields

- Improve interdisciplinary networking (clinical medicine, biology/embryology, material sciences, biochemistry)

- Improvement of collaboration between research and industry
- Invest in multidisciplinarity of the field by bringing researchers from different fields better together

- Challenge: Cross-disciplinary approach

- Biology, engineering, medicine, industry

- Computational biology (data mining, modeling of regulatory pathways)

- Joint conferences, workshops

- Funding mechanisms

\section{Immunologic Understanding and Control}

(5 ideas; 3.5\%)

- Develop conditions for xeno-free defined expansion of ES cells with stable karyotype

- Modulation of the immunologic responses to allogenic cells

- Establish technologies to overcome the rejection of organs and tissue-engineered products

- Establish procedures and protocols that ensure that stem cells and their progeny are well tolerated in allogeneic cell transplants

- Recruit adverse tissue reactivity in a positive direction

\section{Expectation Management/Communication}

(5 ideas; 3.5\%)

- Let's be objective about what we promise

- Not to repeat the mistakes of gene therapy (promise too much)

- Patient demand based on real expectations rather than media hype (Market pull)

- Scientifically honest communication of results vs. media-driven popularistic claims

- Broaden the scope of tissue engineering to include in vivo applications of biomaterials, homing of cells by biomaterials, cell-interactive biomedical devices, for example

Angiogenic Control (4 ideas; $2.8 \%$ )

- Control of angiogenesis

- Achieve histointegration

- Revascularization within ECMs (angiogenesis/arteriogenesis, in vitro and in vivo)

- Achieve rapid vascularization of TE constructs

\section{Additional References: \\ Hoshin methodology}

The Hoshin method used in the preparation of this report is a custom-modified form. However, good descriptions of the classic Hoshin method, along with tools for its execution, can be found at this Web site: http://www.tqe.com/ hoshhdbk.html. 
Of note is that the Hoshin process was successfully used in the planning retreat (1996) to support the development of the first formal NIH request for proposals in tissue engineering in 1997:

RFA: HL-97-005: TISSUE ENGINEERING, BIOMIMETICS, AND MEDICAL IMPLANT SCIENCE, NIH GUIDE, Volume 26, Number 13, April 25, 1997.
Address reprint requests to: Peter C. Johnson, M.D. Scintellix, $L L C$ 1000 Quiet Ridge Circle Raleigh, NC 27614-7257

E-mail: TEeditor@rice.edu 
